Article

\title{
Exercise Training Enhances Platelet Mitochondrial Bioenergetics in Stroke Patients: A Randomized Controlled Trial
}

\author{
Chih-Chin Hsu ${ }^{1,2} \mathbb{D}$, Hsing-Hua Tsai ${ }^{3}$, Tieh-Cheng Fu ${ }^{1}$ and Jong-Shyan Wang ${ }^{1,3,4, *(\mathbb{C})}$ \\ 1 Department of Physical Medicine and Rehabilitation, Keelung Chang Gung Memorial Hospital, Keelung 204, \\ Taiwan; steele0618@gmail.com (C.-C.H.); fic6481@gmail.com (T.-C.F.) \\ 2 School of Medicine, College of Medicine, Chang Gung University, Taoyuan 333, Taiwan \\ 3 Healthy Aging Research Center, Graduate Institute of Rehabilitation Science, College of Medicine, \\ Chang Gung University, Taoyuan 333, Taiwan; serena751004@gmail.com \\ 4 Research Center for Chinese Herbal Medicine, College of Human Ecology, Chang Gung University of \\ Science and Technology, Taoyuan 333, Taiwan \\ * Correspondence: s120011676@gmail.com; Fax: +886-3-2118700
}

Received: 14 November 2019; Accepted: 6 December 2019; Published: 11 December 2019

\begin{abstract}
Exercise training (ET) may impact physical fitness by affecting mitochondrial functions. This study aimed to elucidate the effect of ET on aerobic capacity and platelet mitochondrial bioenergetics (MTB) in stroke patients. Among the 30 stroke patients who underwent the traditional rehabilitation program (TRP), 15 were randomly assigned to have ET ( $50-60 \% \mathrm{VO}_{2 \text { peak }}$ for $30 \mathrm{~min} /$ day, 5 days/week for 4 weeks), and those remaining received only the TRP (control group). The peak exercise capacity $\left(\mathrm{VO}_{2 \text { peak }}\right)$ and platelet MTB, including oxidative phosphorylation (OXPHOS) and the electron transport chain (ETC), were measured through automatic gas analysis and high-resolution respirometry, respectively. The results demonstrated that ET significantly increased the $\mathrm{VO}_{2 \text { peak }}$ $(17.7 \%)$ and $\mathrm{O}_{2}$ uptake efficiency slope (31.9\%) but decreased the ventilation versus $\mathrm{CO}_{2}$ production slope $(-7.65 \%)$. Patients who underwent ET also had significantly enhanced platelet mitochondrial OXPHOS and ETC by activating the FADH2 (Complex II)-dependent pathway, but depressed plasma myeloperoxidase $(-28.4 \%)$ and interleukin-6 levels $(-29.9 \%)$. Moreover, changes in $\mathrm{VO}_{2 \text { peak }}$ levels were positively correlated with changes in platelet OXPHOS and ETC capacities. In conclusion, ET increases the platelet MTB by enhancing Complex II activity in stroke patients. The exercise regimen also enhances aerobic fitness and depresses oxidative stress/pro-inflammatory status in stroke patients.
\end{abstract}

Keywords: stroke; exercise training; platelet; mitochondria

\section{Introduction}

Stroke, caused by cerebral vascular atherosclerosis/thrombosis or intracranial hemorrhage, is the leading cause of long-term disability, which produces enormous global health and economic burdens [1,2]. Stroke-induced functional impairment reduces exercise capacity, which negatively affects the ability of patients to perform their daily activities. This limitation further decreases their independence and quality of life [1,2]. Although a randomized clinical study for 61 chronic stroke patients showed exercise training (ET) improved cardiovascular fitness [3] and a scientific overview encouraged post-stroke survivors to consider the importance of ET in regaining activities of daily living [4], controversy persists regarding how ET influences the hemostasis in stroke patients or stroke prevention [1,5]. 
Platelets play a pivotal role in thrombogenesis of stroke patients [6,7]. Platelet mitochondria are directly involved in the cellular redox balance, activation, and apoptosis, thereby modulating thrombogenesis [8-10]. Regular exercise improves physical performance and aerobic capacity, which is concurrent with reducing the risk of major vascular thrombotic events [11]. According to our previous studies, moderate-intensity ET attenuated platelet reactivity at rest and during strenuous exercise [12,13]. Recent investigations further demonstrated that high-intensity interval training improved the platelet mitochondrial bioenergetics, which might reduce the risk of thrombosis in healthy sedentary individuals [14] and heart failure (HF) patients [15]. However, the effects of ET on platelet mitochondrial bioenergetics in stroke patients have not been extensively reviewed.

A common method to measure mitochondrial bioenergetics is to determine the enzymatic activity of each complex in the electron transport chain (ETC) [16]. However, mitochondria do not work as independent units [17]. Electron transport complexes are interconnected on the mitochondrial inner membrane and turn into respiratory supercomplexes or respirasomes [18]. A polarographic oxygen sensor can measure the mitochondrial respiration of intact cells [19]. Furthermore, individual complexes of the ETC can also be surveyed by addition of exogenous substrates and inhibitor into permeabilized cells [20]. In order to determine the capacities of platelet mitochondria oxidative phosphorylation (OXPHOS) and ETC in stroke patients, the development of novel protocols to evaluate ET effects on mitochondrial OXPHOS and ETC activities are needed.

We hypothesized that ET would improve the hemostasis by enhancing the platelet mitochondria function, which would further result in the increase of exercise capacity in stroke patients. The present study assessed how 4 weeks of moderate-intensity ET (50-60\% of $\mathrm{VO}_{2 \text { peak }}$ ) affected systemic aerobic capacity and platelet mitochondrial oxidative phosphorylation (OXPHOS), as well as ETC activities in stroke patients. With these in-depth analyses, an effective exercise regimen for stroke patients can possibly be established.

\section{Methods}

\subsection{Participants}

A total of 52 stroke patients diagnosed by the neurologist were surveyed from August 2017 to July 2018 in a tertiary care hospital. Brain computed tomography was used to diagnose the hemorrhagic or ischemic stroke. The ischemic stroke subtype was determined according to the TOAST classification system [21]. The inclusion criteria were listed as follows: (I) $\geq 20$ years old; (II) stable stroke events $\geq 3$ months; (III) mini-mental state examination (MMSE) >24; (IV) no acute coronary syndrome; (V) lower extremity function > Brunnstrom stage III with active voluntary movement. Those who had unstable angina, systolic blood pressure $>200 \mathrm{mmHg}$ or diastolic blood pressure $>110 \mathrm{~mm} \mathrm{Hg}$, symptomatic orthostatic hypotension, severe aortic stenosis (peak systolic pressure gradient $>50 \mathrm{mmHg}$, or an aortic valve opening area $<0.75 \mathrm{~cm}^{2}$ ), inflammatory disease within the previous 3 months, uncontrolled cardiac dysrhythmias, uncompensated heart failure (HF), third-degree atrioventricular block, pericarditis or myocarditis within the previous 3 months, embolic disease within the previous 3 months, ST segment displacement $\geq 2 \mathrm{~mm}$ at rest, or uncontrolled diabetes (blood glucose $\geq 300 \mathrm{mg} / \mathrm{dL}$ or $\geq 250 \mathrm{mg} / \mathrm{dL}$ with ketone bodies) were excluded from the study.

All our eligible candidates did not engage in regular physical exercise except 30-45 min of supervised in-hospital traditional rehabilitation programs (TRP), which included balance, neurofacilitation, and therapeutic exercise, as instructed by their rehabilitation physicians, before starting intervention. They were randomly assigned to the intervention (under ET and TRP) and control (under TRP only) groups (Figure S1) based on the computer-generated random allocation sequence. An independent physician performed the randomization procedure, which was concealed to the members performing the intervention and the enrolled participants before starting the experiment. All subjects provided informed consent after the experimental procedures were explained. The Institutional Review 
Board of a tertiary care hospital approved this study (IRB No. 201602058A3) and the clinical trial registration number was NCT03960918.

\subsection{Graded Exercise Test (GET)}

The physician performing the GET was blinded to the subject grouping. He assessed their cardiopulmonary fitness 2 days before and 2 days after 4-week intervention. Moreover, the data collector was isolated from the analytic specialist. Each participant underwent the GET at an incremental work-rate of $10 \mathrm{~W} / \mathrm{min}$ on a bicycle ergometer (Ergoselect 150P, ergoline GmbH, Bitz, Germany) with a continuous monitoring heart rate (HR), brachial blood pressure, and arterial $\mathrm{O}_{2}$ saturation, until the stopping conditions described previously $[13,14] . \mathrm{O}_{2}$ consumption $\left(\mathrm{VO}_{2}\right)$, minute ventilation $\left(\mathrm{V}_{\mathrm{E}}\right)$, and carbon dioxide production $\left(\mathrm{VCO}_{2}\right)$ were measured breath by breath by a cardiopulmonary measurement device (MasterScreen CPX, CareFusion Corp., Hoechberg, Germany), and the $\mathrm{VO}_{2 \text { peak }}$ was defined as the guideline for exercise testing suggested by the American College of Sports Medicine (ACSM) [22].

$\mathrm{V}_{\mathrm{E}}$ and $\mathrm{VCO}_{2}$ responses acquired from the initiation of exercise to the peak values were used to calculate the $\mathrm{V}_{\mathrm{E}}-\mathrm{VCO}_{2}$ slope using least-square linear regression [23]. The $\mathrm{O}_{2}$ uptake efficiency slope (OUES), an estimation of the efficiency of $\mathrm{O}_{2}$ consumption during exercise, was derived from the slope of a natural logarithm plot of $\mathrm{V}_{\mathrm{E}} \mathrm{vs}$. $\mathrm{VO}_{2}$. A greater slope indicated a higher ventilation efficiency $[23,24]$. Additionally, a 6-min walk test $(6 \mathrm{MWT})$ was used to screen functional capacity and exercise endurance in stroke patients [25].

\subsection{Cardiac Hemodynamic Measurements}

A noninvasive continuous cardiac output monitoring system (NICOM, Cheetah Medical, Wilmington, DE, USA) was used to evaluate cardiac hemodynamic response to exercise, which analyzes the phase shift $(\Delta \Phi)$ created by alternating electrical current across the chest of the subject, as described in our previous study [26].

\subsection{Health-Related Quality of Life}

Quality of life (QoL) was measured using a Short Form-36 Health Survey questionnaire (SF-36), the findings of which were related to the co-morbidities of stroke patients [27].

\subsection{Exercise Training (ET) Protocol}

A 30-45 min of TRP was implemented for all included stroke survivors, which was judged by physician discretion. The control subjects only engaged in the TRP. In addition to the TRP, the subjects in the intervention group underwent supervised hospital-based training 20 times ( 5 session/week for 4 weeks) on a bicycle ergometer (Ergoselect 150P, Bitz, Germany) as our previous protocol describes [26]. The ET comprised a warm-up at $30 \%$ of $\mathrm{VO}_{2 \text { peak }}$ for $3 \mathrm{~min}$, followed by continuous $60 \%$ of $\mathrm{VO}_{2 \text { peak }}$ for $30 \mathrm{~min}$, then a cool-down at $30 \%$ of $\mathrm{VO}_{2 \text { peak }}$ for $3 \mathrm{~min}$. The training was terminated when the subject had symptoms/signs suggested by the ACSM guideline [22]. The compliance rate of all our subjects was $100 \%$ (Figure S1).

\subsection{Blood Cell Count}

Based on our previous work [28], an amount of $25 \mathrm{~mL}$ of blood was sampled just prior to each exercise test performed. The first $2 \mathrm{~mL}$ was discarded, then the remaining blood sample was used for the measurements of hematological parameters and platelet function. Each blood sampling was performed after holding medication (anti-platelet or anticoagulant) for $48 \mathrm{~h}$, stroke prevention medication was restarted after the procedure. The blood analysis was performed initially and after 4 weeks of ET. Blood cells were enumerated using a Sysmax SF-3000 cell counter (GMI Inc., Ramsey, $\mathrm{MN}, \mathrm{USA}$ ) and were repetitively analyzed twice to ensure the reproducibility of the results. 


\subsection{Platelet Isolation}

Samples, processed as the above description to avoid platelet activation [28], were collected in polypropylene tubes containing a sodium citrate concentration of $3.8 \mathrm{~g} / \mathrm{dL}$. Platelet rich plasma (PRP) was prepared through centrifugation at $300 \times g$ for $10 \mathrm{~min}$ at approximately $20{ }^{\circ} \mathrm{C}$. Platelets were sedimented through centrifugation of the PRP at $1500 \times g$ for $10 \mathrm{~min}$ at approximately $20^{\circ} \mathrm{C}$ and then were washed once with PBS containing $4 \mathrm{mM}$ ethylenediaminetetraacetic acid (EDTA) (Sigma-Aldrich, St. Louis, MO, USA) to inhibit platelet activation [14,15]. They were mixed with mitochondrial respiration medium (MiR05, containing sucrose $110 \mathrm{mM}$, HEPES $20 \mathrm{mM}$, taurine $20 \mathrm{mM}, \mathrm{K}$-lactobionate $60 \mathrm{mM}, \mathrm{MgCl}_{2} 3 \mathrm{mM}, \mathrm{KH}_{2} \mathrm{PO}_{4} 10 \mathrm{mM}$, EGTA $\left.0.5 \mathrm{mM}, \mathrm{BSA} 1 \mathrm{~g} / \mathrm{L}, \mathrm{pH} 7.1\right)$ to a final concentration of 2 $\times 10^{8}$ cells $/ \mathrm{mL}$. All platelet fractions were analyzed within $2 \mathrm{~h}$ after cell purification.

\subsection{High-Resolution Respirometry}

Platelet mitochondrial respiration was measured by a high-resolution respirometry (Oxygraph-2K, Oroboros Instrument, Innsbruck, Austria) with a stirrer speed of $750 \mathrm{rpm}$ at a constant temperature of $37^{\circ} \mathrm{C}$. Data were acquired and recorded every $2 \mathrm{~s}$ by DatLab software version 6 (Oroboros Instrument, Innsbruck, Austria). Platelets of $2 \times 10^{8}$ cells $/ \mathrm{mL}$ were added to the glass chamber filled with $2 \mathrm{~mL}$ mitochondrial respiration medium (MiR05) for measurement $[14,15]$. The $\mathrm{O}_{2}$ concentration was automatically calculated from barometric pressure and the solubility factor was 0.92 for MiR05.

\subsection{Mitochondrial ETC and OXPHOS in Platelets}

The substrate-uncoupler-inhibitor titration reference protocol (SUIT-RP), consisting of two mitochondrial substrate-controlled experiments (RP1 and RP2), was applied to acquire the platelet mitochondrial bioenergetics (Figure S2A-C). All the chemicals were purchased from the Sigma-Aldrich Corporation.

The SUIT-RP1 was used to measure the mitochondrial ETC capacity (Figure S2A). The cell membrane was permeabilized with digitonin. Malate $(2 \mathrm{mM})$ and pyruvate $(5 \mathrm{mM}), \mathrm{NADH}$-linked (N-linked) substrates were subsequently added $[14,15] . \mathrm{O}_{2}$ consumption was only driven by uncoupling proton leakage (LEAK state, $\mathrm{PM}_{\mathrm{L}}$ ) because the ADP was absent. The OXPHOS capacity $\left(\mathrm{PM}_{\mathrm{P}}\right)$ was then evaluated by $1 \mathrm{mM}$ ADP (Calbiochem, St. Louis, MO, USA) titration. The ETC capacity driven by malate and pyruvate $\left(\mathrm{PM}_{\mathrm{E}}\right)$ was obtained by mitochondrial protonophore carbonyl cyanide-p-trifluoromethoxyphenylhydrazone (FCCP) titration $(0.5 \mu \mathrm{M} / \mathrm{step})$ until no further respiration increased. Glutamate $(10 \mathrm{mM})$ was added to access the maximal ETC capacity driven by NADH or mitochondrial complex I (CI)-related resources $\left(\mathrm{MPG}_{\mathrm{E}}\right)$. Thereafter, $10 \mathrm{mM}$ succinate was used to complete the convergent input of CI and complex II (CII) $\left(\mathrm{MPGS}_{\mathrm{E}}\right)$. Octanoyl-carnitine (Oct) titration $(0.5 \mathrm{mM})$ was performed to evaluate the additional effect of fatty acid oxidation (FAO) (MPGSOct $t_{\mathrm{E}}$ ). The N-linked substrate-dependent respiration and FAO pathways $\left(\mathrm{S}_{\mathrm{E}}\right)$ were blocked by $0.5 \mu \mathrm{M}$ rotenone ( $\mathrm{CI}$ inhibitor). The additional contribution of the mitochondrial complex of glycerophosphate dehydrogenase $(\mathrm{CGpDH})$ was measured with $10 \mathrm{mM}$ glycerophosphate $\left(\mathrm{SG}_{\mathrm{E}}\right)$. At last, the mitochondrial respiration was inhibited by $2.5 \mu \mathrm{M}$ antimycin $\mathrm{A}$, the mitochondrial complex III inhibitor (Figure S2A).

The mitochondrial ETC capacities in the permeabilized platelets were obtained from the following equations:

$$
\begin{gathered}
\text { ETC }_{\mathrm{CI}}\left(\mathrm{PMG}_{\mathrm{E}}\right)=\text { Pyruvat }+ \text { Malate }+\mathrm{ADP}+\mathrm{FCCP}+\text { Glutamate } \\
\mathrm{ETC}_{\mathrm{CI}+\mathrm{CII}}\left(\mathrm{PMGS}_{\mathrm{E}}\right)=\text { Pyruvate }+ \text { Malate }+\mathrm{ADP}+\mathrm{FCCP}+\text { Glutamate + Succinate } \\
\mathrm{ETC}_{\mathrm{CI}+\mathrm{CII}+\mathrm{FAO}}\left(\mathrm{PMGSOct}_{\mathrm{E}}\right)=\text { Pyruvat }+ \text { Malate }+\mathrm{ADP}+\mathrm{FCCP}+\text { Glutamate + Succinate + Oct } \\
\mathrm{ETC}_{\mathrm{CII}}\left(\mathrm{S}_{\mathrm{E}}\right)=\text { Pyruvate }+ \text { Malate }+\mathrm{ADP}+\mathrm{FCCP}+\text { Glutamate }+ \text { Succinate + Oct + Rotenone }
\end{gathered}
$$




$$
\begin{gathered}
\mathrm{ETC}_{\mathrm{CII}+\mathrm{Gp}}\left(\mathrm{SGp}_{\mathrm{E}}\right)=\text { Pyruvate }+ \text { Malate }+\mathrm{ADP}+\mathrm{FCCP}+\text { Glutamate }+ \text { Succinate }+ \text { Oct } \\
\text { + Rotenone + Glycerophosphate }
\end{gathered}
$$

The SUIT-RP2 was to measure the ATP synthase-dependent OXPHOS state (Figure S2B). Following routine respiration and digitonin permeabilization, $1 \mathrm{mM}$ ADP was titrated to accelerate the depletion of residual endogenous substrates. Then, $0.5 \mathrm{mM} \mathrm{OC}$ and $0.1 \mathrm{mM}$ malate were added to evaluate the FAO. An amount of $2 \mathrm{mM}$ Malate was used to reduce the flux from succinate dehydrogenase. This procedure could also activate the mitochondrial malic enzyme and led to higher $\mathrm{O}_{2}$ consumption $\left(\mathrm{OctM}_{\mathrm{P}}\right)$. An amount of $5 \mathrm{mM}$ pyruvate $\left(\mathrm{OctMP}_{\mathrm{P}}\right)$ and $10 \mathrm{mM}$ glutamate $\left(\mathrm{OctMPG}_{\mathrm{P}}\right)$ were sequentially titrated to acquire the N-linked respiration. A consecutive titration of $10 \mathrm{mM}$ succinate and $10 \mathrm{mM}$ glycerophosphate stimulated the mitochondria respiration through CII (OctMPGS $)$ and CGpDH (OctMPGSGP $p_{P}$ ) input. To evaluate the limitation of the OXPHOS system, FCCP $(0.5 \mu \mathrm{M} / \mathrm{step})$ was titrated to access the maximal ETC capacity (OctMPGSGp $\mathrm{p}_{\mathrm{E}}$ ). An amount of $0.5 \mu \mathrm{M}$ rotenone was added to reach the identical SGp state in the RP1 and RP2 protocols. Antimycin A $(2.5 \mu \mathrm{M})$ was added in the final step to block the whole mitochondria respiration.

The capacities of mitochondrial OXPHOS in permeabilized platelets were calculated from the following equations (Figure S2B):

$$
\begin{gathered}
\text { OXPHOS }_{\mathrm{FAO}}\left(\text { Oct }_{\mathrm{P}}\right)=\mathrm{ADP}+\text { Oct } \\
\text { OXPHOS }_{\mathrm{FAO}+\mathrm{CI}}\left(\mathrm{OctMPG}_{\mathrm{P}}\right)=\mathrm{ADP}+\text { Oct }+ \text { Malate }+ \text { Pyruvate + Glutamate } \\
\text { OXPHOS }_{\mathrm{FAO}+\mathrm{CI}+\mathrm{CII}}\left(\mathrm{OctMPGS}_{\mathrm{P}}\right)=\mathrm{ADP}+\text { Oct }+ \text { Malate }+ \text { Pyruvate }+ \text { Glutamate }+ \text { Succinate }
\end{gathered}
$$

This study defined the maximal capacities of OXPHOS and ETC in platelets as the OctMPGSG $\mathrm{p}_{P}$ and OctMPGSGp $\mathrm{E}_{\mathrm{E}}$ states in the RP2 protocol, respectively (Figure S2B).

$$
\begin{gathered}
\mathrm{OXPHOS}_{\mathrm{FAO}+\mathrm{CI}+\mathrm{CII}+\mathrm{Gp}}(\text { OctMPGSGp })=\mathrm{ADP}+\text { Oct }+ \text { Malate }+ \text { Pyruvate } \\
+ \text { Glutamate }+ \text { Succinate }+ \text { Glycerophosphate } \\
\mathrm{ETC}_{\mathrm{FAO}+\mathrm{CI}+\mathrm{CII}+\mathrm{Gp}}\left(\begin{array}{l}
\text { OctMPGSGp } \\
\text { + Succinate }+ \text { Glycerophosphate }+\mathrm{FCCP}
\end{array}\right.
\end{gathered}
$$

\subsection{Biomarkers of Oxidative Stress and Pro-Inflammation in Plasma}

A 5-mL blood sample was placed in a cold centrifuge tube containing $4 \mathrm{mM}$ EDTA, and was immediately centrifuged at $3000 \times g$ for $10 \mathrm{~min}$ at $4{ }^{\circ} \mathrm{C}$. Plasma myeloperoxidase (MPO) (Immunology Consultants Lab., Newberg, OR, USA) and interleukin-6 (IL-6) (eBioscience, SanDiego, CA, USA) levels were quantified by commercially available ELISA kits [26]. Prominent increases of $\mathrm{TNF}_{\alpha}$ and IL-1 occur within few hours in the inflammatory cascade and may be inadequate for the study for chronic stroke patients [29]. Therefore, IL-6 and MPO were reviewed in the study because they have been reported to be associated with chronic low-grade inflammation and good responses to exercise related anti-inflammatory effects $[29,30]$.

\subsection{Statistical Analysis}

Data were expressed as mean \pm SEM and were analyzed using the statistical software package StatView. The experimental results were analyzed by 2 (groups) $\times 2$ (time sample points; i.e., preand post-interventions) repeated measures ANOVA. Bonferroni's post hoc was used to compare cardiopulmonary fitness, biomarkers of plasma oxidative stress and inflammation, and platelet mitochondria functions at the beginning of this study and after 4 weeks of observation in both groups. Pearson correlation analysis was used to determine the correlation between the $\mathrm{VO}_{2 \text { peak }}$ and platelet mitochondrial OXPHOS and ETC in stroke patients. $p$ values lower than 0.05 were considered statistically significant. 


\section{Results}

\subsection{Cardiopulmonary Fitness and Health-Related QoL}

A total of 30 subjects completed the study in the intervention $(n=15)$ and control $(n=15)$ groups (Figure 1). No adverse vascular or thrombotic event occurred in the two groups during the investigation. Differences of baseline anthropometric and clinical parameters between the two groups were not significant (Table 1). Among the included subjects, baseline clinical information and comorbidities of the hemorrhagic stroke patients in the two groups is shown in the Table S1. All our hemorrhagic stroke patients suffered from hypertension. The initial cardiopulmonary fitness of the two groups was also similar (Table 2).

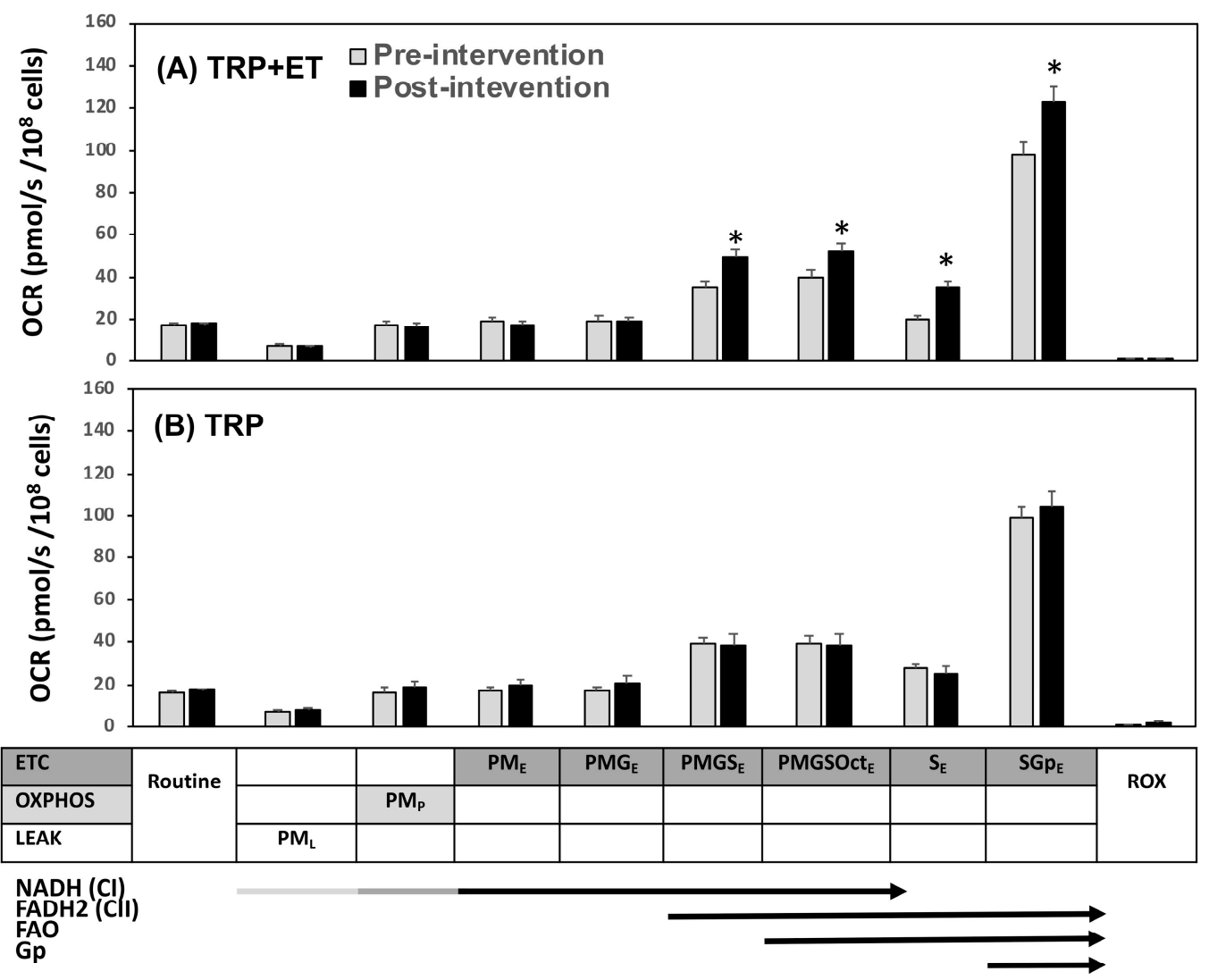

Figure 1. Effects of traditional rehabilitation program (TRP) with exercise training (ET) $(\mathbf{A}, \mathrm{TRP}+\mathrm{ET})$ and without ET (B, TRP) in platelet mitochondrial $\mathrm{O}_{2}$ consumption rate $(\mathrm{OCR})$ using the reference protocol 1 (RP1) protocol in patients with cerebral vascular accident. P, pyruvate; M, malate; G, glutamate; S, succinate; Oct, octanoyl-carnitine; Gp, glycerophosphate; Rot, rotenone; Ama, antimycin; ROX, residual $\mathrm{O}_{2}$ consumption; OXPHOS or $\mathrm{P}$, oxidative phosphorylation; ETC or $\mathrm{E}$, electron transport system; LEAK or $\mathrm{L}$, uncoupling proton leakage. Pre-intervention vs. post-intervention, ${ }^{*} p<0.05$. Values were mean \pm SEM. 
Table 1. Demographic and clinical characteristics in stroke patients.

\begin{tabular}{|c|c|c|c|c|c|}
\hline & & \multicolumn{2}{|c|}{ TRP + ET } & \multicolumn{2}{|c|}{ TRP } \\
\hline & & Pre & Post & Pre & Post \\
\hline \multicolumn{6}{|c|}{ Anthropometrics/Clinical Characteristics } \\
\hline Gender & $n(\mathrm{M} / \mathrm{F})$ & $15(12 / 3)$ & $15(12 / 3)$ & $15(13 / 2)$ & $15(13 / 2)$ \\
\hline Age & year & $55.7 \pm 3.0$ & - & $57.8 \pm 3.9$ & - \\
\hline Height & $\mathrm{cm}$ & $165.7 \pm 1.4$ & $165.7 \pm 1.4$ & $166.8 \pm 1.5$ & $166.8 \pm 1.5$ \\
\hline Weight & $\mathrm{kg}$ & $70.7 \pm 2.7$ & $70.0 \pm 2.6$ & $71.6 \pm 2.8$ & $72.2 \pm 2.9$ \\
\hline BMI & $\mathrm{kg} / \mathrm{m}^{2}$ & $25.8 \pm 1.0$ & $25.6 \pm 0.9$ & $25.7 \pm 1.0$ & $25.9 \pm 1.2$ \\
\hline Heart rate & bpm & $76 \pm 5$ & $73 \pm 6$ & $77 \pm 6$ & $76 \pm 7$ \\
\hline Systolic blood pressure & $\mathrm{mmHg}$ & $135 \pm 10$ & $132 \pm 9$ & $137 \pm 9$ & $136 \pm 8$ \\
\hline $\begin{array}{l}\text { Diastolic blood pressure } \\
\text { Etiology }\end{array}$ & $\mathrm{mmHg}$ & $78 \pm 4$ & $75 \pm 6$ & $81 \pm 5$ & $80 \pm 6$ \\
\hline Ischemia & $n(\%)$ & $12(80)$ & - & $12(80)$ & - \\
\hline Small vessel thrombosis & & $7(47)$ & & $8(53)$ & \\
\hline Embolic & & $5(33)$ & & $4(27)$ & \\
\hline Hemorrhage & $n(\%)$ & $3(20)$ & - & $3(20)$ & - \\
\hline Stroke duration & month & $21 \pm 3$ & - & $23 \pm 4$ & - \\
\hline \multicolumn{6}{|c|}{ Brunnstrom Stage } \\
\hline Stage III & $n(\%)$ & $3(25)$ & - & $4(27)$ & - \\
\hline$>$ Stage III & $n(\%)$ & $12(75)$ & - & $11(73)$ & - \\
\hline \multicolumn{6}{|c|}{ Mini-Mental State Examination } \\
\hline & score & $28.7 \pm 2.9$ & - & $29.2 \pm 4.5$ & - \\
\hline \multicolumn{6}{|c|}{ Risk Factors } \\
\hline Smoking & $n(\%)$ & $6(40)$ & & $6(40)$ & \\
\hline Hyperlipidemia & $n(\%)$ & $12(80)$ & - & $11(73)$ & - \\
\hline Hypertension & $n(\%)$ & $3(20)$ & - & $4(27)$ & - \\
\hline CVD & $n(\%)$ & $7(47)$ & - & $8(53)$ & - \\
\hline Diabetes mellitus & $n(\%)$ & $10(67)$ & - & $9(60)$ & - \\
\hline \multicolumn{6}{|c|}{ Hematological Parameters } \\
\hline Erythrocyte & $10^{6} / \mu \mathrm{L}$ & $5.00 \pm 0.12$ & $5.17 \pm 0.14$ & $4.91 \pm 0.11$ & $4.90 \pm 0.16$ \\
\hline Hemoglobin & $\mathrm{g} / \mathrm{dL}$ & $14.3 \pm 0.4$ & $14.8 \pm 0.5$ & $14.2 \pm 0.4$ & $14.2 \pm 0.7$ \\
\hline Hematocrit & $\%$ & $44.6 \pm 1.3$ & $45.7 \pm 1.4$ & $44.3 \pm 1.3$ & $43.1 \pm 1.9$ \\
\hline Leukocyte & $10^{3} / \mu \mathrm{L}$ & $7.01 \pm 0.21$ & $6.72 \pm 0.40$ & $7.17 \pm 0.26$ & $6.37 \pm 0.53$ \\
\hline Platelet & $10^{3} / \mu \mathrm{L}$ & $264 \pm 13$ & $239 \pm 16$ & $274 \pm 15$ & $255 \pm 12$ \\
\hline \multicolumn{6}{|c|}{ Plasma Oxidative Stress and Pro-Inflammatory Status } \\
\hline MPO & $\mathrm{ng} / \mathrm{mL}$ & $11.70 \pm 0.64$ & $8.38 \pm 1.05 *$ & $11.10 \pm 0.98$ & $12.15 \pm 0.82$ \\
\hline IL-6 & $\mathrm{pg} / \mathrm{mL}$ & $13.69 \pm 1.75$ & $9.61 \pm 1.75^{*}$ & $13.09 \pm 1.84$ & $13.03 \pm 1.73$ \\
\hline \multicolumn{6}{|c|}{ Medicines } \\
\hline ASA & $n(\%)$ & $7(47)$ & $7(47)$ & $8(53)$ & $8(53)$ \\
\hline NOAC & $n(\%)$ & $5(33)$ & $5(33)$ & $4(27)$ & $4(27)$ \\
\hline $\begin{array}{l}\text { HMG CoA reductase } \\
\text { inhibitor }\end{array}$ & $n(\%)$ & $12(80)$ & $12(80)$ & $11(73)$ & $11(73)$ \\
\hline$\beta$-blockers & $n(\%)$ & $3(20)$ & $3(20)$ & $4(27)$ & $4(27)$ \\
\hline ACEI/ARB & $n(\%)$ & $3(20)$ & $3(20)$ & $4(27)$ & $4(27)$ \\
\hline
\end{tabular}

Values are mean \pm SEM. ACEI/ARB, angiotensin converting enzyme inhibitor/angiotensin II receptor blocker; ASA, acetylsalicylic acid; BMI, body mass index; CVD, cardiovascular diseases; ET, exercise training; HMG CoA, 3-hydroxy-3-methyl- glutaryl coenzyme A; IL-6, interleukin-6; MPO, myeloperoxidase; NOAC, non-vitamin K antagonist oral anticoagulant; Pre, pre-intervention; Post, post-intervention; TRP, traditional rehabilitation program. * estimated by repeated measured ANOVA. 
Table 2. The effect of exercise training on cardiopulmonary responses to exercise and health-related quality of life in stroke patients.

\begin{tabular}{|c|c|c|c|c|c|}
\hline & & \multicolumn{2}{|c|}{ TRP + ET } & \multicolumn{2}{|c|}{ TRP } \\
\hline & & Pre & Post & Pre & Post \\
\hline \multicolumn{6}{|c|}{ Peak Exercise Performance } \\
\hline Work-rate & watt & $81.9 \pm 4.8$ & $100.0 \pm 6.1^{*}$ & $78.8 \pm 7.8$ & $80.5 \pm 6.9$ \\
\hline Hear rate & bpm & $132 \pm 4$ & $139 \pm 3^{*}$ & $133 \pm 4$ & $134 \pm 5$ \\
\hline Stroke volume & $\mathrm{mL}$ & $68.1 \pm 3.2$ & $80.5 \pm 4.1^{*}$ & $70.8 \pm 3.5$ & $75.7 \pm 3.7$ \\
\hline Cardiac output & $\mathrm{L} / \mathrm{min}$ & $8.97 \pm 0.41$ & $11.16 \pm 0.79 *$ & $9.42 \pm 0.52$ & $10.15 \pm 0.91$ \\
\hline $\mathrm{V}_{\mathrm{E}}$ & $\mathrm{L} / \mathrm{min}$ & $50.3 \pm 3.1$ & $62.5 \pm 3.7^{*}$ & $51.2 \pm 4.1$ & $53.4 \pm 5.2$ \\
\hline $\mathrm{VO}_{2}$ & $\mathrm{~mL} / \mathrm{min}$ & $1155 \pm 80$ & $1359 \pm 85$ * & $1165 \pm 76$ & $1194 \pm 65$ \\
\hline $\mathrm{VCO}_{2}$ & $\mathrm{~mL} / \mathrm{min}$ & $1291 \pm 87$ & $1590 \pm 97$ * & $1348 \pm 92$ & $1395 \pm 95$ \\
\hline MET & score & $4.7 \pm 0.2$ & $5.5 \pm 0.2 *$ & $4.6 \pm 0.3$ & $4.8 \pm 0.2$ \\
\hline OUES & unit & $630 \pm 38$ & $831 \pm 58 *$ & $634 \pm 61$ & $575 \pm 36$ \\
\hline $\mathrm{V}_{\mathrm{E}}-\mathrm{VCO}_{2}$ slope & unit & $35.3 \pm 1.9$ & $32.6 \pm 2.2$ & $34.6 \pm 1.9$ & $34.3 \pm 1.5$ \\
\hline 6-min walk test & meter & $325.4 \pm 76.2$ & $380.4 \pm 47.4$ * & $320.5 \pm 62.1$ & $315.6 \pm 56.3$ \\
\hline \multicolumn{6}{|c|}{ Short Form-36 Health Survey Questionnaire } \\
\hline Physical & score & $40.1 \pm 1.7$ & $44.2 \pm 2.0^{*}$ & $41.5 \pm 2.1$ & $40.6 \pm 2.2$ \\
\hline Mental & score & $36.1 \pm 2.1$ & $44.9 \pm 2.2 *$ & $36.8 \pm 2.2$ & $34.8 \pm 2.7$ \\
\hline
\end{tabular}

Following 4 weeks of interventions, the stroke patients underwent ET displayed significantly increased work-rate, heart rate, stroke volume (SV), cardiac output (CO) as well as, $\mathrm{V}_{\mathrm{E}}, \mathrm{VCO}_{2}, \mathrm{OUES}$, $\mathrm{VO}_{2 \text { peak, }}$ and $6 \mathrm{MWT}$ distance $(p<0.05)$. In addition, a significant decrease of $\mathrm{V}_{\mathrm{E}}-\mathrm{VCO}_{2}$ slope $(p<0.05)$ was detected in stroke patients with ET. However, significant changes in cardiopulmonary fitness were not identified in those with 4 weeks of traditional rehabilitation training. In our observation, the TRP did not influence indicators of ventilatory efficiency and functional capacity in stroke patients. Detailed information is shown in Table 2.

In health-related QoL, 4 weeks of ET significantly increased the subclass scores of the physical (scores from 40.1 to $44.2, p<0.05$ ) and mental (scores from 36.1 to $44.9, p<0.05$ ) dimensions (Table 2). However, QoL in the stroke patients who underwent 4 weeks of TRP remained unchanged (Table 2).

\subsection{Hematologic Parameters and Oxidative Stress/Inflammatory Biomarkers}

There were no significant changes in hemograms (i.e., erythrocyte, hemoglobin, hematocrit, leukocyte, and platelet) following 4 weeks of TRP with or without ET (Table 1). However, ET associated with TRP considerably reduced the plasma levels of MPO $(p<0.05)$ and IL-6 $(p<0.05)$. The oxidative stress and pro-inflammation in plasma were unchanged following the TRP alone (Table 1).

\subsection{Mitochondrial ETC and OXPHOS in Platelets}

Neither TRP + ET nor TRP alone changed the routine respiration and uncoupling proton leakage of platelets (Figures 1 and 2). Moreover, the two therapeutic regimens were also insufficient to influence platelet ETC driven by NADH (CI)-dependent pathway $\left(\mathrm{PM}_{\mathrm{E}}\right.$ and $\mathrm{PMG}_{\mathrm{E}}$, Figure $\left.1 \mathrm{~A}, \mathrm{~B}\right)$. The OXPHOS driven by NADH (CI)- and FAO-dependent pathways $\left(\mathrm{Oct}_{\mathrm{p}}, \mathrm{OctM}_{\mathrm{p}}, \mathrm{OctMP}_{\mathrm{p}}\right.$, and OctMPG $\left.\mathrm{G}_{\mathrm{p}}\right)$ were not affected (Figure 2A,B). 

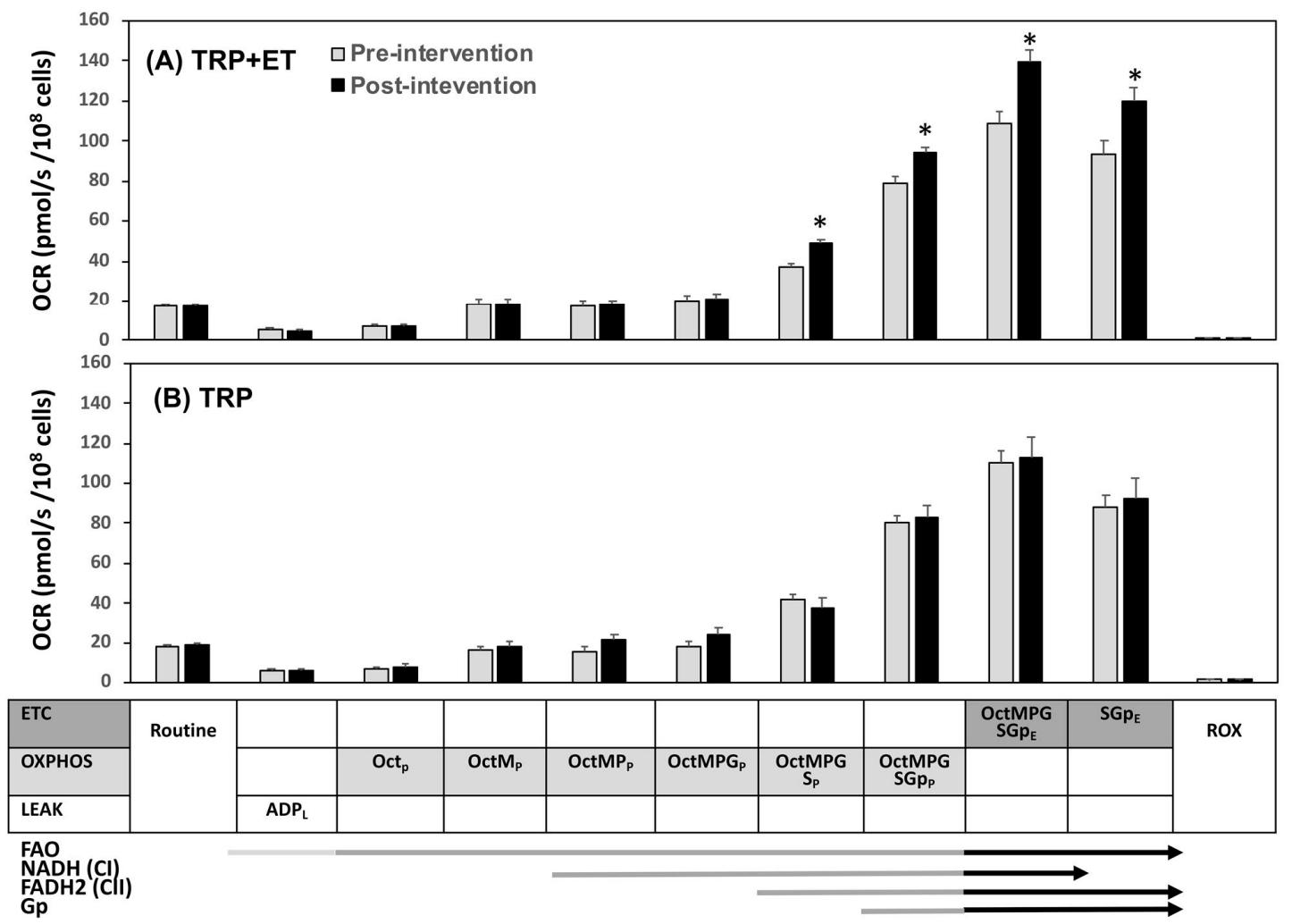

Figure 2. Effects of TRP with (A, TRP + ET) and without (B, TRP) exercise training in platelet mitochondrial $\mathrm{O}_{2}$ consumption rate (OCR) using the reference protocol 2 (RP2) protocol in patients with cerebral vascular accident. P, pyruvate; M, malate; G, glutamate; S, succinate; Oct, octanoyl-carnitine; $\mathrm{Gp}$, glycerophosphate; Rot, rotenone; Ama, antimycin; $\mathrm{ROX}$, residual $\mathrm{O}_{2}$ consumption; $\mathrm{OXPHOS}$ or $\mathrm{P}$, oxidative phosphorylation; ETC or ${ }_{\mathrm{E}}$, electron transport system; LEAK or $\mathrm{L}$, uncoupling proton leakage. Pre-intervention vs. post-interve ntion, ${ }^{*} p<0.05$. Values were mean $\pm \mathrm{SEM}$.

Notably, TRP + ET for 4 weeks significantly increased platelet flavin adenine dinucleotide (FADH2) in CII-related ETC levels $\left(\right.$ PMGS $_{\mathrm{E}}$, PMGSOct $_{\mathrm{E}}, \mathrm{S}_{\mathrm{E}}$, and $\mathrm{SG}_{\mathrm{E}}$ ) (Figure $1 \mathrm{~A}, p<0.05$ ). Moreover, changes in $\mathrm{VO}_{2 \text { peak }}$ levels were directly related to changes of PMGS (Figure $3 \mathrm{~A}, \mathrm{r}=0.587, p<0.001$ ), PMGSOct $_{\mathrm{E}}$ (Figure 3B, $\mathrm{r}=0.581, p<0.001$ ), $\mathrm{S}_{\mathrm{E}}$ (Figure $3 \mathrm{C}, \mathrm{r}=0.650, p<0.001$ ), and $\mathrm{SGp}_{\mathrm{E}}$ (Figure 3D, $\mathrm{r}=0.846$, $p<0.001)$.

On the other hand, TRP + ET also enhanced succinate-involved platelet OXPHOS levels (Figure 2A, $p<0.05)$, such as OctMPGS , rather than OctMPGP. Furthermore, the exercise regimen also augmented the capacities of the maximal OXPHOS (OctMPGSGp,$p<0.05$ ) and ETC (OctMPGSGp,$p<0.05$ ) in platelet (Figure 2A). Additionally, changes in $\mathrm{VO}_{2 \text { peak }}$ levels were positively associated with changes

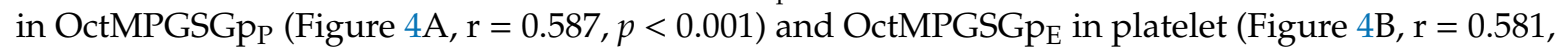
$p<0.001)$. However, no significant changes in platelet mitochondrial bioenergetics, including ETC and OXPHOS capacities, were observed in the control subjects (Figures 1 and 2). 


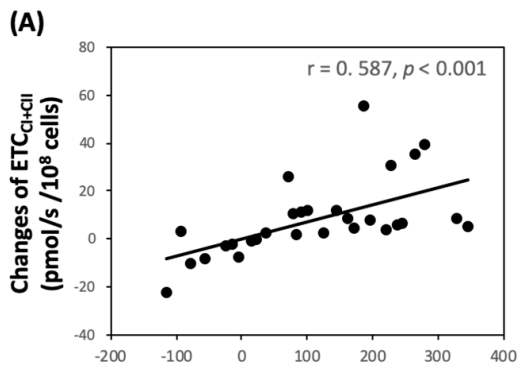

(B)

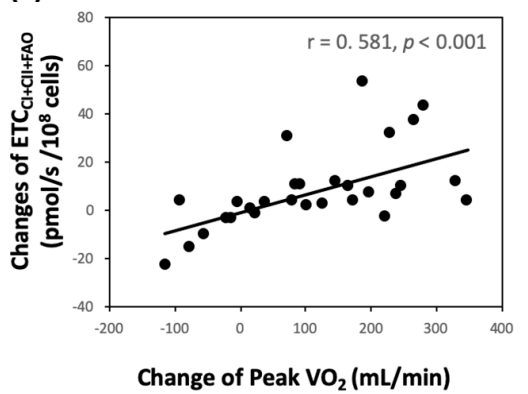

(C)

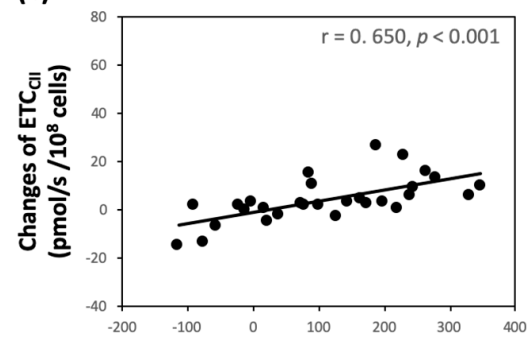

(D)

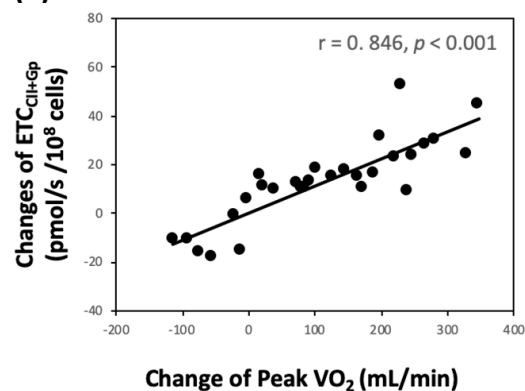

Figure 3. Correlations between changes of aerobic capacity $\left(\mathrm{VO}_{2 p e a k}\right)$ and platelet mitochondrial electron transport chain (ETC) in patients with cerebral vascular accident. (A) ETC $\mathrm{CI}+\mathrm{CII}\left(\mathrm{PMGS}_{\mathrm{E}}\right)=$ Pyruvate + Malate + ADP + FCCP + Glutamate + Succinate; $($ B $)$ ETC $_{\mathrm{CI}+\mathrm{CII}+\mathrm{FAO}}\left(\right.$ PMGSOct $\left._{\mathrm{E}}\right)=$ Pyruvate + Malate + ADP + FCCP + Glutamate + Succinate + Octanoyl-Carnitine; $($ C $)$ ETC $_{\mathrm{CII}}\left(\mathrm{S}_{\mathrm{E}}\right)=$ Pyruvate + Malate + ADP + FCCP + Glutamate + Succinate + Octanoyl-Carnitine + Rotenone; $($ D) ETC CII $+\mathrm{Gp}$ $\left(\mathrm{SGp}_{\mathrm{E}}\right)=$ Pyruvate + Malate $+\mathrm{ADP}+\mathrm{FCCP}+$ Glutamate + Succinate + Octanoyl-Carnitine + Rotenone + Glycerophosphate.
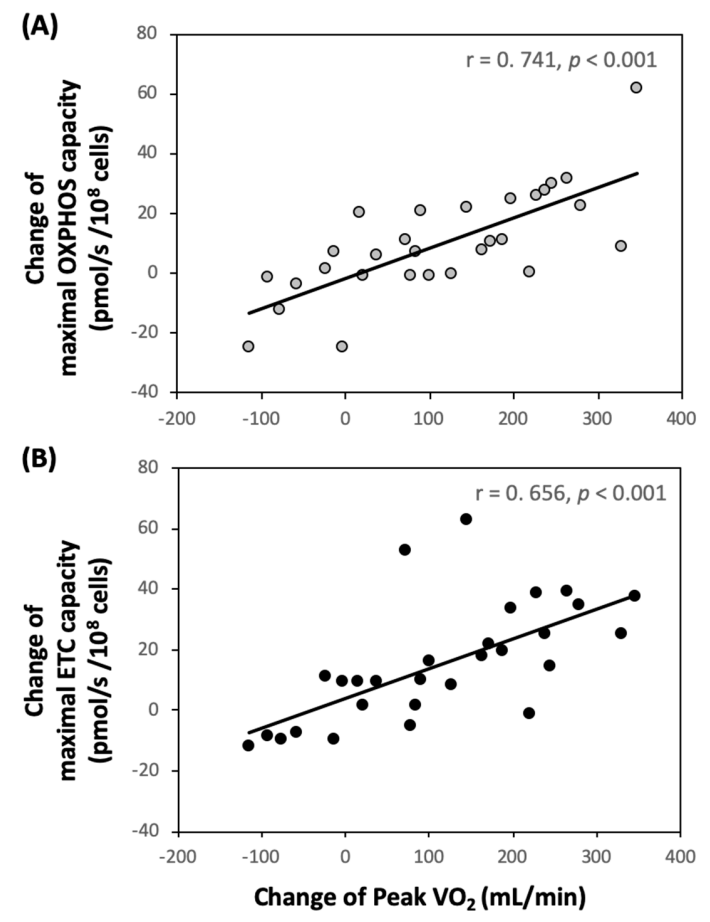

Figure 4. Correlations between changes of aerobic capacity $\left(\mathrm{VO}_{2 p e a k}\right)$ and the capacities of maximal OXPHOS and ETC in platelets (as the OctMPGSGp P $_{P}$ and OctMPGSGp $\mathrm{p}_{\mathrm{E}}$ states using RP2 protocol, respectively) in patients with cerebral vascular accident. (A) OXPHOS $\mathrm{FAO}+\mathrm{CI}+\mathrm{CII}+\mathrm{Gp}$ (OctMPGSGp $)$ = ADP + Octanoyl-Carnitine + Malate + Pyruvate + Glutamate + Succinate + Glycerophosphate; (B) $\mathrm{ETC}_{\mathrm{FAO}+\mathrm{CI}+\mathrm{CII}+\mathrm{Gp}}($ OctMPGSGp $)=\mathrm{ADP}+$ Octanoyl-carnitine + Malate + Pyruvate + Glutamate + Succinate + Glycerophosphate + FCCP. 


\section{Discussion}

The present work exhibited that 4 weeks of moderate-intensity ET improved exercise capacity and ventilatory/hemodynamic efficiency, and physiological adaptation was accompanied by reduced oxidative stress and pro-inflammatory status in stroke patients. The ET-induced relief of oxidative stress in both ischemic and hemorrhagic stroke patients in the study might reverse the systemic coagulopathy and may be helpful in ischemic and hemorrhagic stroke prevention. In addition to the above interesting clinical findings, two novel reference protocols (SUIT-RP1 and SUIT-RP2) were developed in this study to determine the platelet mitochondrial OXPHOS and ETC capacities. To our knowledge, this human study is the first to demonstrate that moderate-intensity ET effectively enhanced platelet mitochondrial OXPHOS and ETC capacities through increasing Complex II activity in stroke patients.

\subsection{Exercise Capacity}

In comparison with healthy individuals of the same age, reduced exercise capacity in stroke patients, especially about one month after onset of stroke, decreased their ability to perform daily activity independently, thereby further worsening their QoL [4,5]. The ventilatory parameters obtained from the GET may convey information regarding prognosis of circulatory disorders [23]. The $\mathrm{VO}_{2 \text { peak }}$ and OUES are indicators of cardiorespiratory fitness and $\mathrm{O}_{2}$ metabolic efficiency, respectively [22,23]. The $\mathrm{V}_{\mathrm{E}}-\mathrm{VCO}_{2}$ slope has long been known to be a useful survival predictor in patients with circulatory disorders [23]. Our previous study reported that these indices of ventilatory efficiency were correlated with exercise-induced central and peripheral hemodynamic changes in HF patients. We also found that ET effectively improved ventilation-perfusion matching during exercise, and this improvement was accompanied by better QoL in HF patients [25]. In this study, $\mathrm{VO}_{2 \text { peak }}$ and OUES, as well as the cardiac pumping capability (such as $\mathrm{CO}$ and SV) of stroke patients, improved significantly after 4 weeks of moderate-intensity ET. These post-exercise benefits associated with a decreased trend in the $\mathrm{V}_{\mathrm{E}}-\mathrm{VCO}_{2}$ slope suggest that ET effectively improves ventilatory and hemodynamic efficiencies, leading to an increase of exercise capacity in stroke patients. In addition, the exercise regimen also increased the capacities of the maximal platelet OXPHOS and ETC in stroke patients. Moreover, improved $\mathrm{VO}_{2 \text { peak }}$ was positively correlated with platelet mitochondrial bioenergetics in the stroke patients. Hence, platelet mitochondrial bioenergetics may be an ideal surrogate for determination of systemic aerobic capacity in stroke patients.

\subsection{Mitochondrial Functionalities in Platelets}

Emerging findings indicate that neuron death in stroke patients is associated with an impaired mitochondria respiratory capacity, which may be caused by the inability to maintain energy homeostatic to compensate for the acute cerebrovascular event. Oxygen deprivation after stroke onset contribute to mitochondria dysfunction, resulting in overproduction of reactive oxidative species (ROS) [31]. Systemic inflammatory response occurs subsequently and further changes of pro- or anti-inflammatory cytokines are associated with the severity of neurological deficit [32]. Additionally, mitochondria play a pivotal role in platelet-mediated aggregation and are essential in non-ATP- mediated thrombosis $[8,9]$. Hence, platelet mitochondrial bioenergetics can be a marker reflecting the oxidative stress of stroke.

Since mitochondria are highly sensitive and respond dynamically to oxidative stress in their microenvironment [33], mitochondria dysfunctions are frequently observed in the early stage of a typical ischemia/reperfusion tissue injury, such as acute myocardial infarction or stroke. Overproduction of mitochondria-derived ROS has been considered to be the consequence of the interaction of the malfunctional respiratory chain with $\mathrm{O}_{2}$ during reperfusion [34]. The present investigation demonstrated that moderate-intensity ET significantly decreased plasma MPO and IL-6 levels, inflammatory cytokines, in stroke patients. Thus, it is considered that the ET may blunt stroke-induced oxidative stress possibly incited by mitochondrial dysfunction. 
The accumulation of intracellular succinate after an ischemic insult, resulting in elevated mitochondrial ROS production, has been reported [35]. Oxidative stress may further induce succinate overproduction by decreasing succinate dehydrogenase (SDH) activity [36]. Our previous study on sedentary healthy young adults found that ET under $\mathrm{O}_{2}$ tension of $12 \%$ for 30 min significantly enhanced platelet mitochondria SDH activity and Complex II-related respiration [14]. In another clinical investigation, we further identified that ET elevated the platelet mitochondrial $\mathrm{O}_{2}$ consumption rate against oxidative stress by increasing Complex II activity in HF patients [14]. In the present study, 4 weeks of ET enhanced platelet mitochondrial OXPHOS and ETC capacities by increasing Complex II activity, but lowered plasma MPO and IL-6 concentrations in the stroke patients. In this investigation, we further detected that an improved aerobic capacity was positively correlated with changes in FADH2 (CII)-related OXPHOS and ETC activities. Therefore, ET-induced elevation in platelet Complex II activity may rapidly eliminate succinate and further reduce ROS production from platelet mitochondria. This ET-induced physiologic adaptation may eventually depress circulatory oxidative stress and pro-inflammatory status in stroke patients.

\subsection{Health-Related Quality of Life}

In addition to an increase in aerobic capacity, this study demonstrated that ET significantly improved mental health in stroke patients. These findings imply that the moderate-intensity exercise regimen effectively facilitated the ability of patients to cope with the physical demands of daily activity, subsequently improving psychosocial status in stroke patients. Furthermore, an improved health-related QoL might improve survival probability in stroke patients and simultaneously reduce the financial burden in their health care system [1,2].

\subsection{Limitations of This Study}

Although limited stroke patients were included in the study, the results regarding platelet mitochondrial bioenergetics have high values of statistical power ranging from 0.902 to 1.000 (Table S2). Additionally, this study mainly focused on the effects of ET on platelet mitochondrial bioenergetics rather than platelet reactivity. Our previous studies investigated the effect of different aerobic exercise regimens on underlying mechanisms of platelet reactivity in healthy people and patients with cardiovascular disorders [11-13]. A recent study further reported that aerobic exercise training markedly suppressed the hypoxia-induced oxidative damage of platelet mitochondria and consequently, reduced platelet-mediated thrombin generation in healthy sedentary individuals [14]. However, the role of ET-mediated improvement of platelet mitochondrial function on platelet reactivity and coagulation in stroke patients requires further investigation.

Six hemorrhagic strokes, 3 in each group, suffered from hypertension in this study. It is well-known that hypertension is highly prevalent and an important risk factor for hemorrhagic stroke [37]. Increased platelet aggregation has been observed in aneurysmal subarachnoid hemorrhagic patients and was possibly related with abnormal platelet ADP secretion [38]. Moreover, elevated oxidative stress may impair mitochondria function and could be a potent trigger of consumptive coagulopathy to result in abnormal cerebral hemostasis [39]. Therefore, we recruited hemorrhagic stroke patients because both hypertension and abnormal platelet function can be expected in them.

\section{Conclusions}

In the present study, 4 weeks of ET improved systemic aerobic capacity through increased ventilatory and hemodynamic efficiencies in stroke patients. The exercise regimen also increased the capacities of OXPHOS and ETC by enhancing mitochondrial Complex II activity in platelets. These experimental findings may provide insight into the identification of adequate exercise regimen to increase physical performance and improve platelet mitochondrial bioenergetics in stroke patients. 
Supplementary Materials: The following are available online at http://www.mdpi.com/2077-0383/8/12/2186/s1, Figure S1: Flowchart of enrolled stroke patients during following-up, Figure S2: Graphs showing the reference protocols of substrate-uncoupler-inhibitor titration and convergent electron transfers in platelets, Table S1: Baseline demographics in hemorrhagic stroke patients, Table S2: Estimated power in measured parameters.

Author Contributions: Conceptualization, J.-S.W.; Data curation, C.-C.H., H.-H.T., J.-S.W., and T.-C.F.; Formal analysis, H.-H.T. and J.-S.W.; Funding acquisition, C.-C.H., T.-C.F., and J.-S.W.; Methodology, H.-H.T. and T.-C.F.; Review and editing, C.-C.H. and J.-S.W.; Writing-original draft, C.-C.H., H.-H.T., and J.-S.W. All authors critically revised the manuscript for important intellectual content and approved the final manuscript.

Funding: This work was supported by the National Science Council of Taiwan (grant number NSC 106-2314-B-182-048-MY3), Keelung Chang Gung Medical Research Program (grant number CMRPD3I0021 and CMRPG2G0011), and Healthy Aging Research Center, Chang Gung University (grant number EMRPD1A0841).

Acknowledgments: We would like to thank Pei-Hsun Yuan in the Department of Physical Medicine and Rehabilitation, Keelung Chang Gung Memorial Hospital, for collecting clinical information. This manuscript has undergone English language editing by Martyn Rittman of MDPI.

Conflicts of Interest: No conflicts of interest, financial or otherwise, are declared by the authors.

\section{References}

1. Meschia, J.F.; Bushnell, C.; Boden-Albala, B.; Braun, L.T.; Bravata, D.M.; Chaturvedi, S.; Creager, M.A.; Eckel, R.H.; Elkind, M.S.V.; Fornage, M.; et al. Guidelines for the primary prevention of stroke: A statement for healthcare professionals from the American Heart Association/American Stroke Association. Stroke 2014, 45, 3754-3832. [CrossRef] [PubMed]

2. Feigin, V.L.; Lawes, C.M.; Bennett, D.A.; Anderson, C.S. Stroke epidemiology: A review of population-based studies of incidence, prevalence, and case-fatality in the late 20th century. Lancet Neurol. 2003, 2, 43-53. [CrossRef]

3. Macko, R.F.; Ivey, F.M.; Forrester, L.W.; Hanley, D.; Sorkin, J.D.; Katzel, L.I.; Silver, K.H.; Goldberg, A.P. Treadmill exercise rehabilitation improves ambulatory function and cardiovascular fitness in Patients with chronic stroke: A randomized, controlled trial. Stroke 2005, 36, 2206-2211. [CrossRef] [PubMed]

4. Han, P.; Zhang, W.; Kang, L.; Ma, Y.; Fu, L.; Jia, L.; Yu, H.; Chen, X.; Hou, L.; Wang, L. Clinical evidence of exercise benefits for stroke. Adv. Exp. Med. Biol. 2017, 1000, 131-151.

5. Olney, S.J.; Nymark, J.; Brouwer, B.; Culham, E.; Day, A.; Heard, J.; Henderson, M.; Parvataneni, K. A randomized controlled trial of supervised versus unsupervised exercise programs for ambulatory stroke survivors. Stroke 2006, 37, 476-481. [CrossRef]

6. Kotzailias, N.; Finsterer, J.; Zellner, M.; Marsik, C.; Dukic, T.; Jilma, B. Platelet function in mitochondriopathy with stroke and stroke-like episodes. Thromb. Haemost. 2004, 91, 544-552.

7. Milionis, H.; Liontos, A.; Vemmos, K.; Spengos, K. Antiplatelet treatment in stroke: New insights. Curr. Pharm. Des. 2016, 22, 4617-4626. [CrossRef]

8. Garcia-Souza, L.F.; Oliveira, M.F. Mitochondria: Biological roles in platelet physiology and pathology. Int. J. Biochem. Cell Biol. 2014, 50, 156-160. [CrossRef]

9. Zharikov, S.; Shiva, S. Platelet mitochondrial function: From regulation of thrombosis to biomarker of disease. Biochem. Soc. Trans. 2013, 41, 118-123. [CrossRef]

10. Kramer, P.A.; Chacko, B.K.; Ravi, S.; Johnson, M.S.; Mitchell, T.; Darley-Usmar, V.M. Bioenergetics and the oxidative burst: Protocols for the isolation and evaluation of human leukocytes and platelets. J. Vis. Exp. 2014, 85, e51301. [CrossRef]

11. Wang, J.S. Exercise prescription and thrombogenesis. J. Biomed. Sci. 2006, 13, 753-761. [CrossRef] [PubMed]

12. Wang, J.S.; Jen, C.J.; Chen, H.I. Effects of exercise training and deconditioning on platelet function in men. Arterioscler. Thromb. Vasc. Biol. 1995, 15, 1668-1674. [CrossRef] [PubMed]

13. Wang, J.S.; Li, Y.S.; Chen, J.C. Effects of exercise training and deconditioning on platelet aggregation induced by alternating shear stress in men. Arterioscler. Thromb. Vasc. Biol. 2005, 25, 454-460. [CrossRef] [PubMed]

14. Wu, L.H.; Chang, S.C.; Fu, T.C.; Huang, C.H.; Wang, J.S. High-intensity interval training improves mitochondrial function and suppresses thrombin generation in platelets undergoing hypoxic stress. Sci. Rep. 2017, 7, e4191. [CrossRef] [PubMed] 
15. Chou, C.H.; Fu, T.C.; Tsai, H.H.; Hsu, C.C.; Wang, C.H.; Wang, J.S. High-intensity interval training enhances mitochondrial bioenergetics of platelets in patients with heart failure. Int. J. Cardiol. 2019, 274, 214-220. [CrossRef]

16. Wiedemann, F.R.; Vielhaber, S.; Schröder, R.; Elger, C.E.; Kunz, W.S. Evaluation of methods for the determination of mitochondrial respiratory chain enzyme activities in human skeletal muscle samples. Anal. Biochem. 2000, 279, 55-60. [CrossRef]

17. Picard, M.; Taivassalo, T.; Ritchie, D.; Wright, K.J.; Thomas, M.M.; Romestaing, C.; Hepple, R.T. Mitochondrial structure and function are disrupted by standard isolation methods. PLoS ONE 2011, 6, e18317. [CrossRef]

18. Letts, J.A.; Fiedorczuk, K.; Sazanov, L.A. The architecture of respiratory supercomplexes. Nature 2016, 537, 644-648. [CrossRef]

19. Chacko, B.K.; Kramer, P.A.; Ravi, S.; Benavides, G.A.; Mitchell, T.; Dranka, B.P.; Ferrick, D.; Singal, A.K.; Ballinger, S.W.; Bailey, S.M.; et al. The Bioenergetic Health Index: A new concept in mitochondrial translational research. Clin. Sci. (Lond.) 2014, 127, 367-373. [CrossRef]

20. Pesta, D.; Gnaiger, E. High-resolution respirometry: OXPHOS protocols for human cells and permeabilized fibers from small biopsies of human muscle. Methods Mol. Biol. 2012, 810, 25-58.

21. Marnane, M.; Duggan, C.A.; Sheehan, O.C.; Merwick, A.; Hannon, H.; Curtin, D.; Harris, D.; Williams, E.B.; Horgan, G.; Kyne, L.; et al. Stroke Subtype classification to mechanism-specific and undetermined categories by TOAST, A-S-C-O, and causative classification system: Direct comparison in the North Dublin population stroke study. Stroke 2010, 41, 1579-1586. [CrossRef] [PubMed]

22. American College of Sports Medicine. General principle of exercise prescription. In ACSM's Guidelines for Exercise Testing and Prescription; Thompson, W.R., Gordon, N.F., Pescatello, L.S., Eds.; Lippincott Williams \& Wilkins: Philadelphia, PA, USA, 2010; pp. 152-182.

23. Arena, R.; Guazzi, M.; Myers, J. Ventilatory abnormalities during exercise in heart failure: A mini review. Curr. Respir. Med. Rev. 2007, 3, 179-187. [CrossRef]

24. Hollenberg, M.; Tager, I.B. Oxygen uptake efficiency slope: An index of exercise performance and cardiopulmonary reserve requiring only submaximal exercise. J. Am. Coll. Cardiol. 2000, 36, 194-201. [CrossRef]

25. ATS Committee on Proficiency Standards for Clinical Pulmonary Function Laboratories. ATS statement: Guidelines for the six-minute walk test. Am. J. Respir. Crit. Care Med. 2002, 166, 111-117. [CrossRef] [PubMed]

26. Fu, T.C.; Wang, C.H.; Lin, P.S.; Hsu, C.C.; Cherng, W.J.; Huang, S.C.; Liu, M.H.; Chiang, C.L.; Wang, J.S. Aerobic interval training improves oxygen uptake efficiency by enhancing cerebral and muscular hemodynamics in patients with heart failure. Int. J. Cardiol. 2013, 167, 41-50. [CrossRef]

27. Lai, S.M.; Perera, S.; Duncan, P.W.; Bode, R. Physical and social functioning after stroke: Comparison of the Stroke Impact Scale and Short Form-36. Stroke 2003, 34, 488-493. [CrossRef]

28. Wang, J.S.; Jen, C.J.; Kung, H.C.; Lin, L.J.; Hsiue, T.R.; Chen, H.I. Different effects of strenuous exercise and moderate exercise on platelet function in men. Circulation 1994, 90, 2877-2885. [CrossRef]

29. Petersen, A.M.; Pedersen, B.K. The anti-inflammatory effect of exercise. J. Appl. Physiol. 2005, 98, 1154-1162. [CrossRef]

30. Khan, A.A.; Alsahli, M.A.; Rahmani, A.H. Myeloperoxidase as an active disease biomarker: Recent bioehcmical and pathological perspectives. Med. Sci. 2018, 6, 33. [CrossRef]

31. Liu, F.; Lu, J.; Manaenko, A.; Tang, J.; Hu, Q. Mitochondria in ischemic stroke: New insight and implications. Aging Dis. 2018, 9, 924-937. [CrossRef]

32. Basic Kes, V.; Simundic, A.M.; Nikolac, N.; Topic, E.; Demarin, V. Pro-inflammatory and anti-inflammatory cytokines in acute ischemic stroke and their relation to early neurological deficit and stroke outcome. Clin. Biochem. 2008, 41, 1330-1334. [CrossRef] [PubMed]

33. Carter, H.N.; Chen, C.C.; Hood, D.A. Mitochondria, muscle health, and exercise with advancing age. Physiology (Bethesda) 2015, 30, 208-223. [CrossRef] [PubMed]

34. Anzell, A.R.; Maizy, R.; Przyklenk, K.; Sanderson, T.H. Mitochondrial quality control and disease: Insights into ischemia-reperfusion injury. Mol. Neurobiol. 2018, 55, 2547-2564. [CrossRef] [PubMed]

35. Mills, E.; O’Neill, L.A. Succinate: A metabolic signal in inflammation. Trends Cell. Biol. 2014, 24, 313-320. [CrossRef] [PubMed] 
36. Chouchani, E.T.; Pell, V.R.; Gaude, E.; Aksentijević, D.; Sundier, S.Y.; Robb, E.L.; Logan, A.; Nadtochiy, S.M.; Ord, E.N.J.; Smith, A.C.; et al. Ischaemic accumulation of succinate controls reperfusion injury through mitochondrial ROS. Nature 2014, 515, 431-435. [CrossRef] [PubMed]

37. Woo, D.; Haverbusch, M.; Sekar, P.; Kissela, B.; Khoury, J.; Schneider, A.; Kleindorfer, D.; Szaflarski, J.; Pancioli, A.; Jauch, E.; et al. Effect of untreated hypertension on hemorrhagic stroke. Stroke 2004, 35, 1703-1708. [CrossRef]

38. Perez, P.; Lukazewicz, A.C.; Lenck, S.; Nizard, R.; Drouet, L.; Payen, D. Platelet activation and aggregation after aneurysmal subarachnoid hemorrhage. BMC Neurol. 2018, 18, 57. [CrossRef]

39. Chen, Y.W.; Chen, Y.C.; Fu, T.C.; Wang, C.H.; Huang, S.C.; Wang, J.S. Anemic comorbidity reduces capacity of endogenous thrombin generation and is associated with consumptive coagulopathy in patients with heart failure. Int. J. Cardiol. 2013, 168, 4965-4967. [CrossRef]

(C) 2019 by the authors. Licensee MDPI, Basel, Switzerland. This article is an open access article distributed under the terms and conditions of the Creative Commons Attribution (CC BY) license (http://creativecommons.org/licenses/by/4.0/). 